
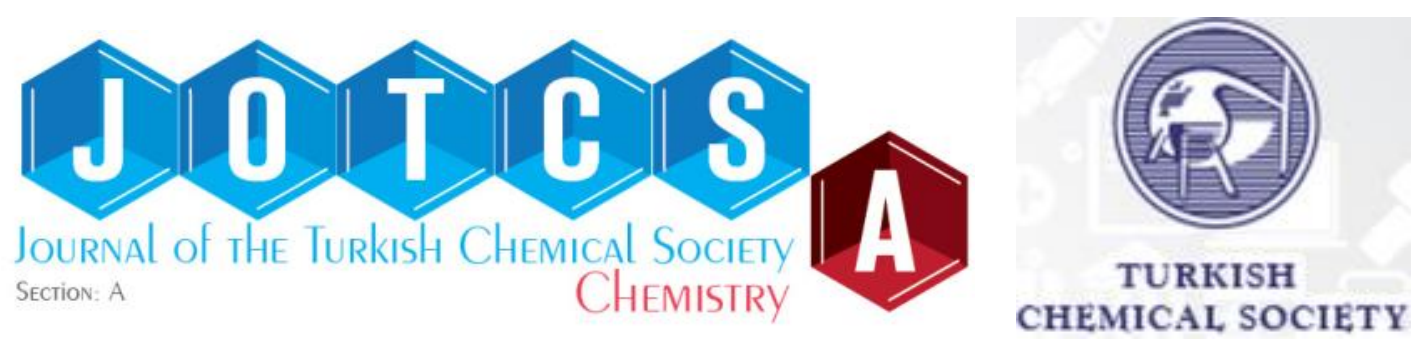

\title{
Synthesis, E/Z isomerization, and antimicrobial studies of different structured novel ketone derivatives
}

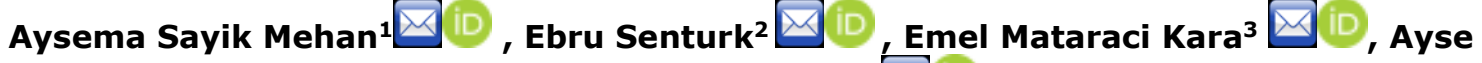 Serguzel Yusufoglu $1 * \Delta$}

1 Istanbul University-Cerrahpasa, Engineering Faculty, Chemistry Department, Organic Chemistry Division, Avcilar-Istanbul, Turkey

2 Istanbul University-Cerrahpasa, Institute of Science, Chemistry Division, Avcilar-Istanbul, Turkey

3 Istanbul University-, Pharmacy Faculty, Pharmaceutical Microbiology Department, BeyazitTurkey

Abstract: In this study, five novel ketones' (k1- k5), five new hydrazones (h1-h5) and five new semicarbazones (s1-s5) were synthesized. The synthesized compounds were identified and their $E / Z$ isomerization were studied by $\mathrm{FT}-\mathrm{IR},{ }^{1} \mathrm{H}-\mathrm{NMR},{ }^{13} \mathrm{C}-\mathrm{NMR}$, mass spectrometric and chromatographic methods. These mentioned hydrazones and semicarbazones were investigated for their antimicrobial activities. Seven bacterial species three fungal species were tested. Ciprofloxacin and fluconazole were used as standard compounds. The MIC values were determined. The relationship between structure and antimicrobial actvity was reported. It was found that hydrazones exhibited better activity than that of semicarbazones. Besides, acetylacetone as a diketone yielded the known 1-(2,4-dinitrophenyl)-3,5-dimethyl-1H-pyrazole (p1) which showed higher antimicrobial activity than hydrazones and semicarbazones against Klebsiella pneumoniae ATCC 4352, Proteus mirabilis ATCC 14153, Staphylococcus epidermidis ATCC 12228, Enterococcus faecalis ATCC 29212, Candida parapsilosis ATCC 22019, and Candida tropicalis ATCC 750.

Keywords Diazo compounds; Substituent effect; Heterocycles; Antimicrobial activity; Pyrazole

Submitted: November 21, 2018. Accepted: April 04, 2019.

Cite this: Sayik Mehan A, Senturk E, Mataraci Kara E, Serguzel Yusufoglu A. Synthesis, E/Z isomerization, and antimicrobial studies of different structured novel ketone derivatives. JOTCSA. 2019;6(2):177-88.

DOI: https://dx.doi.org/10.18596/jotcsa.486487.

*Corresponding author. E-mail: ayseserg@istanbul.edu.tr.

\section{INTRODUCTION}

Ketones and their derivatives are valuable compounds in organic synthesis. Among them, hydrazones and semicarbazones have many applications in organic, analytic, and medicinal chemistry (1-3). They can form stable complexes with transition metal ions, protect and purify carbonyl compounds by making them highly stable (4). Due to the literature survey many hydrazones and semicarbazones show biological activities such as antimicrobial, anticonvulsant (5), analgesic, antiinflammatory, antitubercular, antitumor properties (6), pesticide effcets and are plant growth regulators (7). With the aim to obtain new antimicrobial agents, novel five hydrazones (h1-h5) (Table 2) and five semicarbazones (s1-s5) (Table 3) were synthesized by starting from their corresponding ketones (k1- k5) (Table 1). k1-k4 were obtained by Friedel Crafts 
acylation. Three of them are original. Tolyl undecyl ketone, bromophenyl undecyl ketone, and chlorophenyl undecyl ketone are original ones. According to the literature, all the synthesized hydrazones (h1-h5) and semicarbazones are novel compounds. Hydrazones includes azomethine group which enables the formation of pyrazoles. 1-(2,4dinitrophenyl)-3,5-dimethyl-1H pyrazole was synthesized because pyrazoles and substituted pyrazoles have considerable biological importance as being anticancer, anti-inflammatory, anticonvulsant, antiviral and antiprotozoal (8-9).
The above mentioned new hydrazones, semicarbazones and pyrazole (Table 4) (10) were characterized by FT-IR, ${ }^{1} \mathrm{H}-\mathrm{NMR},{ }^{13} \mathrm{C}-$ NMR, mass and chromatographic methods. Their E/Z isomerization was analyzed. Their antimicrobial activities were tested against seven species of bacteria and three species of fungi by using ciprofloxacin and fluconazole as standards. Their MIC values were determined. These studies let us to examine the relationship between structure and antimicrobial activity. Hydrazones were more active than semicarbazones. 1-(2,4dinitrophenyl)-3,5-dimethyl-1H pyrazole was tested against more and different microbials in this study and found as the most effective compound (11-12).

Table 1 Ketones as starting compounds.

\begin{tabular}{ll}
\hline Ketone's code & Full name \\
\hline k1 & Phenyl undecyl ketone \\
k2 & Tolyl undecyl ketone \\
k3 & Bromophenyl undecyl ketone \\
k4 & Chlorophenyl undecyl ketone \\
k5 & Cyclopropyl phenyl ketone \\
\hline
\end{tabular}

Table 2 Synthesized hydrazones.

\begin{tabular}{ll}
\hline $\begin{array}{l}\text { Hydrazone's } \\
\text { code }\end{array}$ & Full name \\
\hline h1 & Phenyl undecyl ketone 2,4-dinitrophenylhydrazone \\
h2 & Tolyl undecyl ketone 2,4-dinitrophenylhydrazone \\
h3 & Bromophenyl undecyl ketone 2,4-dinitrophenylhydrazone \\
h4 & Chlorophenyl undecyl ketone 2,4-dinitrophenylhydrazone \\
h5 & Cyclopropyl phenyl ketone 2,4-dinitrophenylhydrazone \\
\hline
\end{tabular}

Table 3 Synthesized semicarbazones.

\begin{tabular}{ll}
\hline Semicarbazone'code & Full name \\
\hline s1 & Phenyl undecyl ketone semicarbazone \\
s2 & Tolyl undecyl ketone semicarbazone \\
s3 & Bromophenyl undecyl ketone semicarbazone \\
s4 & Chlorophenyl undecyl ketone semicarbazone \\
s5 & Cyclopropyl phenyl undecyl ketone semicarbazone \\
\hline
\end{tabular}

Table 4 Synthesized pyrazole.

\begin{tabular}{ll}
\hline $\begin{array}{l}\text { Pyrazole's } \\
\text { code }\end{array}$ & Full name \\
\hline p1 & 1-(2,4-dinitrophenyl)-3,5-dimethyl-1H pyrazole \\
\hline
\end{tabular}

\section{MATERIALS AND METHODS}

\section{Chemicals and Devices}

Chemicals were supplied from Merck and Aldrich. Reactions' statuses were monitored by TLC (silica gel $60 \mathrm{~F}_{254}$, n-hexane/EtOAc, $1: 1)$

FT-IR data were obtained by using an ATR type Bruker Vertex 70 spectrometer. The NMR spectra were recorded at $500 \mathrm{MHz}$ for
${ }^{1} \mathrm{H}$ and $125 \mathrm{MHz}$ for ${ }^{13} \mathrm{C}$ using $\mathrm{Me}_{4} \mathrm{Si}$ as the internal standard in $\mathrm{CDCl}_{3}$ or DMSO. GC-MS were recorded on Shimadzu QP2010 Plus. A Buchi melting point B-540 apparatus was used for melting point determinations.

\section{Alkyl and phenyl substituted ketone synthesis \\ Phenyl undecyl ketone (k1), Tolyl undecyl ketone (k2), Bromophenyl undecyl ketone (k3) and Chlorophenyl undecyl ketone (k4)}


were synthesized by Friedel Crafts acylation as follows; $56 \mathrm{mmol}$ benzene for $\mathrm{k} 1$; toluene for $k 2$; bromobenzene for $k 3$; chlorobenzene for $\mathrm{k} 4$; and $19 \mathrm{mmol}$ anhydrous $\mathrm{AlCl}_{3}$ were cooled on an ice bath. $19 \mathrm{mmol}$ dodecanoic acid chloride was added from a separatory funnel. $\mathrm{HCl}$ discharging was completed in an hour by heating on a hot water bath. Benzene phase was rinsed with $\mathrm{NaOH}$ and water and then dried over $\mathrm{MgSO}_{4}$. The ketone was purified on column chromatography with acetone /petroleum ether (1:9) (13). Cyclopropyl phenyl ketone (k5) was purchased and used for the synthesis of h5-s5, and acetyl acetone (k6) was also purchased and used for synthesis of $\mathrm{p} 1$.

\section{Hydrazone and pyrazole synthesis}

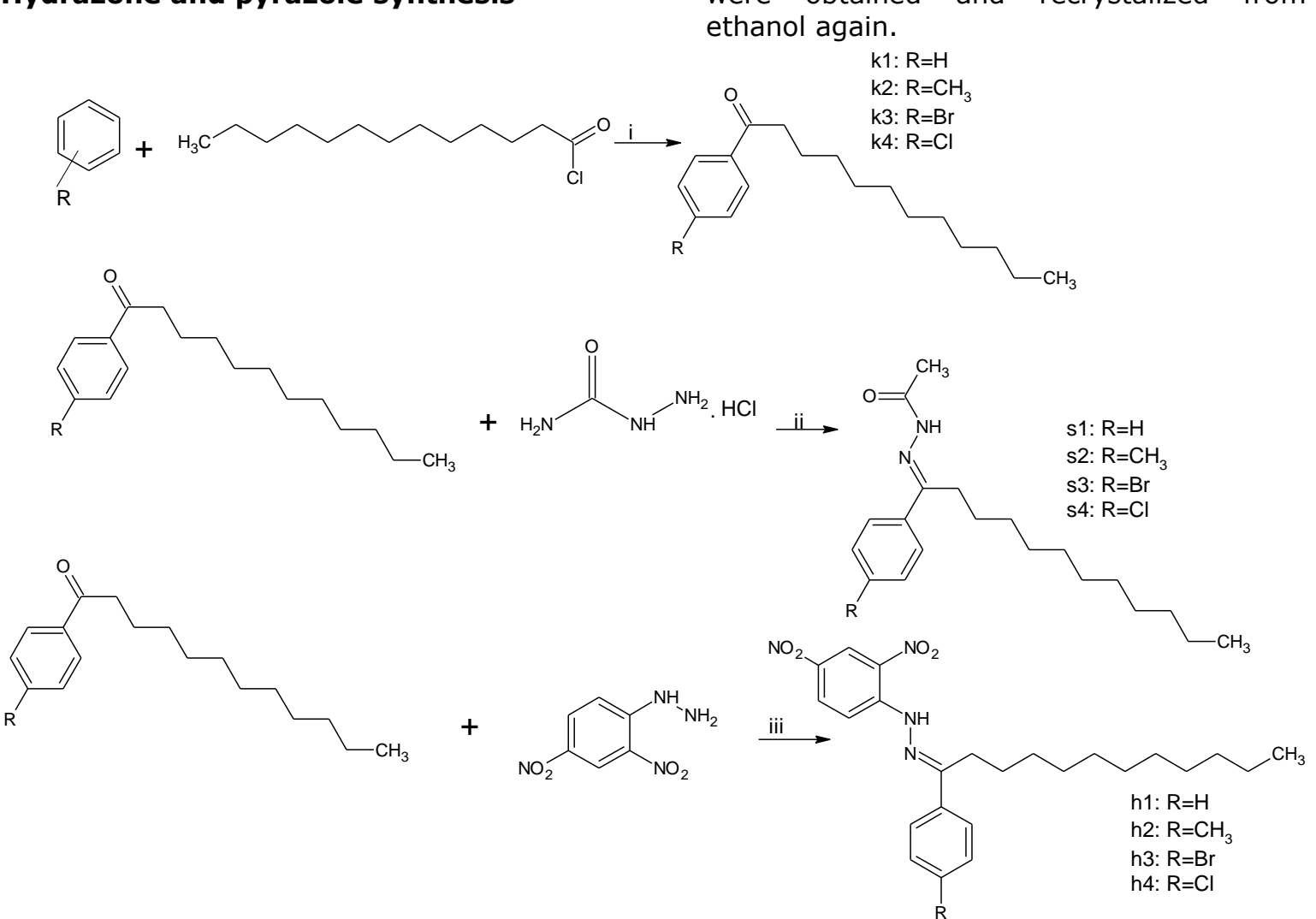

Scheme 1. General overview to the reactions.

$1.0 \mathrm{mmol}$ (gram) ketone and $1.0 \mathrm{mmol} 2,4-$ dinitrophenyl hydrazine were refluxed for 60 $\mathrm{h}$ in $20 \mathrm{~mL}$ of $\mathrm{n}$-propanol. Reaction was monitored by TLC. n-propanol was evaporated from the rotary evaporator. Crude hydrazones (h1-h5) were recrystallized from n-propanol. p1 was also synthesized according to this procedure and recrystallized from methanol.

\section{Semicarbazone synthesis}

$0.5 \mathrm{~g}$ semicarbazide. $\mathrm{HCl}, 0.8 \mathrm{~g} \mathrm{NaOAc}$, and $0.5 \mathrm{~g}$ of ketone were dissolved in $5 \mathrm{~mL}$ of water. $0.5 \mathrm{~mL}$ of ethanol was then added. The mixture was shaken well and kept on a hot water bath for $1 \mathrm{~h}$. The mixture was then cooled to room temperature and poured into the ice-water mixture. Crystals were obtained and recrystalized from ethanol again.

k1: $\mathrm{R}=\mathrm{H}$

k3: $\mathrm{R}=\mathrm{B}$

$\mathrm{R}=\mathrm{CH}_{3}$

s3: $\mathrm{R}=\mathrm{Br}$

s4: $\mathrm{R}=\mathrm{Cl}$

3: $\mathrm{R}=\mathrm{B}$

4: $\mathrm{R}=\mathrm{C}$ 
<smiles>C#CCC#CC</smiles>

k5: Cyclopropyl phenyl ketone<smiles>CC(=O)N/N=C(\c1ccccc1)C1CC1</smiles>

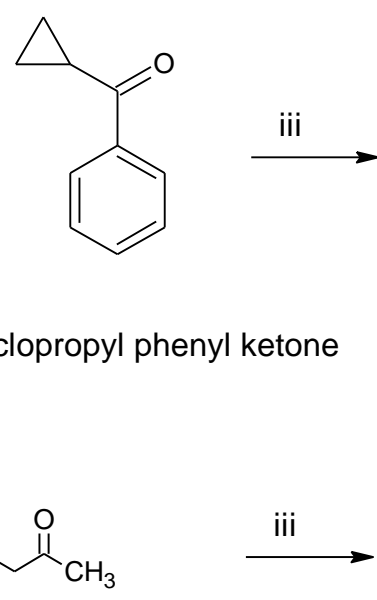

s5: Cyclopropyl phenyl ketone semicarbazone

k5: Cyclopropyl phenyl ketone<smiles>O=[N+]([O-])c1ccc(NN=C(c2ccccc2)C2CC2)c([N+](=O)[O-])c1</smiles>

h5: Cyclopropyl phenyl ketone 2,4-dinitrophenylhydrazone<smiles>Cc1cc(C)n(-c2ccc([N+](=O)[O-])cc2[N+](=O)[O-])n1</smiles>

k6: Acetyl acetone

p1: 1-(2,4-dinitrophenyl)-3,5-dimethyl-1H pyrazole

i) $\mathrm{AlCl}_{3}$ cooled on ice bath; $\mathrm{HCl}$ discharging on hot water bath; rinsed with $\mathrm{NaOH}$; dried over $\mathrm{MgSO}_{4}$.

ii) $\mathrm{NaOAC}, \mathrm{H}_{2} \mathrm{O}$, ethanol on hot water bath for $1 \mathrm{~h}$; cooled to room temperature; poured into ice-water mixture.

iii) n-Propanol, reflux, $60 \mathrm{~h}$

Physical and spectral data of compounds h1-h5, s1-s5, and p1

h1 (Phenyl undecyl ketone 2,4dinitrophenylhydrazone): Orange solid; yield: $343 \mathrm{mg}$, (78 \%); mp:101-102 ${ }^{\circ} \mathrm{C}$; FTIR (ATR): $\bar{u}=3297,2895,2812,1591$, $1554,1468,1287,1245,1098,902,836$, $791 \mathrm{~cm}^{-1} ;{ }^{1} \mathrm{H}-\mathrm{NMR}$ (500 MHz, DMSO): $\delta=0.828 \quad \mathrm{ppm} \quad\left(\mathrm{t}, 3 \mathrm{H}, \mathrm{J}=6.83, \mathrm{CH}_{3}\right)$, $\delta=1.216-1.628 \mathrm{ppm} \quad(\mathrm{m}, 16 \mathrm{H}, \mathrm{J}=7.23$, $\left.\left(\mathrm{CH}_{2}\right)_{8}\right), \delta=1.314 \mathrm{ppm}(\mathrm{p}, 2 \mathrm{H}, \mathrm{J}=6.35$, (E) $\beta$ $\left.\mathrm{CH}_{2}\right), \delta=1.416 \mathrm{ppm}(\mathrm{t}, 2 \mathrm{H}, \mathrm{J}=7.32$, (E) a $\left.\mathrm{CH}_{2}\right), \delta=1.582 \mathrm{ppm}(\mathrm{p}, 2 \mathrm{H}, \mathrm{J}=8.29$, (Z) $\beta$ $\left.\mathrm{CH}_{2}\right), \delta=2.896 \mathrm{ppm}(\mathrm{t}, 2 \mathrm{H}, \mathrm{J}=8.29$, (Z) a $\left.\mathrm{CH}_{2}\right), \quad \delta=6,977-7.479$ ppm (m, alkyl substituted benzene protons), $\delta=8.095 \mathrm{ppm}$ (d, J=9.27, $\mathrm{NO}_{2}$ substituted benzene protons), $\delta=7.930-7.949 \mathrm{ppm}(\mathrm{m}, \mathrm{J}=1.95$, $3.42,1.46,2.93, \mathrm{NO}_{2}$ substituted benzene protons) $\delta=8.899 \mathrm{ppm}(\mathrm{s},(\mathrm{E}), \mathrm{NH})$, $\delta=11.257$ ppm (s, (Z), NH) ${ }^{13} \mathrm{C}-\mathrm{NMR}(500$ $\mathrm{MHz}$, DMSO): $\delta=12.818$ ppm $\left(\mathrm{CH}_{3}\right)$, $\delta=20.961-28.055 \mathrm{ppm}\left(\left(\mathrm{CH}_{2}\right)_{8}\right), \delta=20.168$ ppm (a $\left.\mathrm{CH}_{2}\right), \delta=115.366-143.457$ ppm (aromatic benzene carbons), $\delta=155.418$ - ppm $(\mathrm{C}=\mathrm{N})$; Anal. calcd. for $\mathrm{C}_{24} \mathrm{H}_{31} \mathrm{~N}_{4} \mathrm{O}_{4}$ : C, 65.16; $\mathrm{H}, 7.69 ; \mathrm{N}, 12.67$. Found: $\mathrm{C}, 65.21$; $\mathrm{H}, 7.61 ; \mathrm{N}, 12.63$; MS (m/z): 98, 99, 299, $357,441\left(\mathrm{M}^{+}\right)$

h2 (Tolyl undecyl ketone 2,4dinitrophenylhydrazone): Bright pomegranate flower colored solid; yield: 299 mg, (66 \%); mp: 122-123 ${ }^{\circ} \mathrm{C}$; FT-IR (ATR): $\bar{u}=3318,3110,2921,2851,2287$, 2108, 1613, 1584, 1495, 1416, 1255, $1102,1057,1016,919,848,818 \mathrm{~cm}^{-1} ;{ }^{1} \mathrm{H}-$ NMR (500 MHz, DMSO): $\delta=0.829 \mathrm{ppm}(\mathrm{t}$, $\left.3 \mathrm{H}, \mathrm{J}=7.32, \mathrm{CH}_{3}\right), \delta=1.218-1.630 \mathrm{ppm}(\mathrm{m}$, $\left.16 \mathrm{H}, \mathrm{J}=7.81,\left(\mathrm{CH}_{2}\right)_{8}\right), \delta=1.310 \mathrm{ppm}(\mathrm{t}$, $2 \mathrm{H}, \mathrm{J}=6.83$, (E) a $\mathrm{CH}_{2}$ ), $\delta=1.409 \mathrm{ppm}(\mathrm{p}$, $2 \mathrm{H}, \mathrm{J}=7.32$, (E) $\left.\beta \mathrm{CH}_{2}\right), \delta=1.568 \mathrm{ppm}(\mathrm{p}$, $\left.2 \mathrm{H}, \mathrm{J}=7.81,(\mathrm{Z}) \beta \mathrm{CH}_{2}\right), \delta=2.874 \mathrm{ppm}(\mathrm{t}$, $2 \mathrm{H}, \mathrm{J}=8.29$, (Z) a $\left.\mathrm{CH}_{2}\right), \quad \delta=7.288-7.849$ ppm (d,d, $4 \mathrm{H}, \quad \mathrm{J}=7.81,7.83, \mathrm{CH}_{3}$ substituted aromatic protons), $\delta=8.084$ ppm ( $d, J=9.76$, protons next to the $\mathrm{NH})$, $\delta=8.406 \mathrm{ppm}$ (dd, $\mathrm{J}=2.93,2.44$, protons next to the $\mathrm{NO}_{2}$ ), $\delta=8.899 \mathrm{ppm}$ (sd, $\mathrm{J}=2.44$, proton between two $\mathrm{NO}_{2}$ ), 
$\delta=11.251 \mathrm{ppm}(\mathrm{s}, 1 \mathrm{H}, \mathrm{NH}){ }^{13} \mathrm{C}-\mathrm{NMR}(500$ $\mathrm{MHz}$, DMSO): $\delta=14.625 \mathrm{ppm}\left(\mathrm{CH}_{3}\right)$, $\delta=21.601-29.847 \mathrm{ppm}\left(\left(\mathrm{CH}_{2}\right)_{8}\right), \delta=31.979$ ppm (a $\left.\mathrm{CH}_{2}\right), \delta=117.144-145.250 \mathrm{ppm}$ (aromatic benzene carbons), $\delta=157.325$ ppm $(\mathrm{C}=\mathrm{N})$; Anal. calcd. for $\mathrm{C}_{25} \mathrm{H}_{34} \mathrm{~N}_{4} \mathrm{O}_{4}$ : C, $66.08 ; \mathrm{H}, 7.49 ; \mathrm{N}, 12.33$. Found: $\mathrm{C}, 66.04$; $\mathrm{H}, 7.46 ; \mathrm{N}, 12.37$; MS (m/z):100, 425, 455 $\left(\mathrm{M}^{+}\right)$

h3 (Bromophenyl undecyl ketone 2,4dinitrophenylhydrazone): Bright orange colored solid; yield: $431 \mathrm{mg}$, (83\%); mp: 119-120 ${ }^{\circ} \mathrm{C}$; FT-IR (ATR): $\overline{\mathrm{u}}=3304(\mathrm{NH})$, 2921，2852，1590，1536，1499，1262, $1331,1004,907,836 \mathrm{~cm}^{-1} ;{ }^{1} \mathrm{H}-\mathrm{NMR}$ (500 $\mathrm{MHz}, \mathrm{DMSO}): \delta=0.832 \mathrm{ppm}(\mathrm{t}, 3 \mathrm{H}, \mathrm{J}=6.83$, $\left.\mathrm{CH}_{3}\right), \delta=1.215-1.234 \mathrm{ppm}(\mathrm{m}, 16 \mathrm{H}$, $\left.\mathrm{J}=9.76,\left(\mathrm{CH}_{2}\right)_{8}\right), \delta=1.319 \mathrm{ppm}(\mathrm{t}, 2 \mathrm{H},(\mathrm{E}) \mathrm{a}$ $\left.\mathrm{CH}_{2}\right), \delta=1.418 \mathrm{ppm}(\mathrm{p}, 2 \mathrm{H}, \mathrm{J}=6.83$, (E) $\beta$ $\left.\mathrm{CH}_{2}\right), \delta=1.584 \mathrm{ppm}\left(\mathrm{p}, 2 \mathrm{H},(\mathrm{Z}) \beta \mathrm{CH}_{2}\right)$, $\delta=2.883 \mathrm{ppm}\left(\mathrm{t}, 2 \mathrm{H}, \mathrm{J}=7.81\right.$, (Z) a $\left.\mathrm{CH}_{2}\right)$, $\delta=7.672-7.888 \mathrm{ppm}(\mathrm{d}, \mathrm{d}, 4 \mathrm{H}, \mathrm{J}=8.29,8.79$, $(2 \mathrm{H}, 2 \mathrm{H})$ chlorine substituted aromatic protons), $\delta=8.093 \mathrm{ppm}(\mathrm{d}, 1 \mathrm{H}, \mathrm{J}=9.76$, next to the $\mathrm{NH}), \delta=8.419 \mathrm{ppm}(\mathrm{d}, 1 \mathrm{H}$, $\mathrm{J}=2.44$, next to the $\left.\mathrm{NO}_{2}\right), \delta=8.907 \mathrm{ppm}(\mathrm{s}$, proton between two $\left.\mathrm{NO}_{2}\right), \delta=11.245 \mathrm{ppm}$ (s, $1 \mathrm{H}, \mathrm{NH}){ }^{13} \mathrm{C}-\mathrm{NMR}$ (500 $\left.\mathrm{MHz}, \mathrm{DMSO}\right)$ : $\delta=14.825$ ppm $\left(\mathrm{CH}_{3}\right), \quad \delta=20.968-27.994$ ppm $\left(\left(\mathrm{CH}_{2}\right)_{8}\right), \delta=30.168 \mathrm{ppm}\left(\mathrm{a} \mathrm{CH}_{2}\right)$, $\delta=115.393-143.323 \quad \mathrm{ppm} \quad$ (aromatic benzene carbons), $\delta=154.285-p p m(C=N)$; Anal. calcd. for $\mathrm{C}_{24} \mathrm{H}_{31} \mathrm{BrN}_{4} \mathrm{O}_{4}: \mathrm{C}, 55.49 ; \mathrm{H}$, 5.97; N, 10.79. Found: C, 55.53; H, 5.92; $\mathrm{N}, 10.77$; MS (m/z):180, 351, 420, 442, $520\left(\mathrm{M}^{+}\right)$

h4 (Chlorophenyl undecyl ketone 2,4dinitrophenylhydrazone): Orange colored solid; yield: $374 \mathrm{mg}$, (79 \%); mp: 106-107 ${ }^{\circ} \mathrm{C}$; FT-IR (ATR): $\overline{\mathrm{u}}=3305,3220,3121$, 2920, 2851, 2106, 1614, 1588, 1498, $1491,1420,1330,1305,1261,1132$, $1091,1057,855,835 \mathrm{~cm}^{-1} ;{ }^{1} \mathrm{H}-\mathrm{NMR}(500$ $\mathrm{MHz}, \mathrm{DMSO}): \delta=0.892 \mathrm{ppm}(\mathrm{t}, 3 \mathrm{H}, \mathrm{J}=7.32$, $\left.\mathrm{CH}_{3}\right), \quad \delta=1.279-1.332 \mathrm{ppm}(\mathrm{m}, 16 \mathrm{H}$, $\left.\mathrm{J}=7.81,\left(\mathrm{CH}_{2}\right)_{8}\right), \delta=1.384 \mathrm{ppm}(\mathrm{p}, 2 \mathrm{H}$, $J=7.32$, (E) $\left.\beta \mathrm{CH}_{2}\right), \delta=1.481 \mathrm{ppm}(\mathrm{t}, 2 \mathrm{H}$, $\mathrm{J}=7.81$, (E) a $\left.\mathrm{CH}_{2}\right), \delta=1.630 \mathrm{ppm}(\mathrm{p}, 2 \mathrm{H}$, $\mathrm{J}=7.81$, (Z) $\left.\beta \mathrm{CH}_{2}\right), \delta=2.945 \mathrm{ppm}(\mathrm{t}, 2 \mathrm{H}$, $J=8.29$, (Z) a $\left.\mathrm{CH}_{2}\right), \delta=7.59-7.61 \mathrm{ppm}$ (dd, dd, J=2.93, 1.95,4.88, 2.44, 4H, $(2 \mathrm{H}$, $2 \mathrm{H})$ chlorine substituted aromatic protons), $\delta=8.02 \mathrm{ppm}(\mathrm{d}, 1 \mathrm{H}, \mathrm{J}=1.95$, next to the $\mathrm{NH}), \delta=8.40 \mathrm{ppm}(\mathrm{d}, 1 \mathrm{H}, 2.93$, next to the $\mathrm{NO}_{2}$ ), $\delta=9.01 \mathrm{ppm}$ ( $\mathrm{s}$, proton between two $\left.\mathrm{NO}_{2}\right), \delta=11.25 \mathrm{ppm}(\mathrm{s}, 1 \mathrm{H}, \mathrm{NH}){ }^{13} \mathrm{C}-\mathrm{NMR}$ (500 MHz, DMSO): $\delta=14.622 \mathrm{ppm}\left(\mathrm{CH}_{3}\right)$, $\delta=22.768-29.817 \mathrm{ppm}\left(\left(\mathrm{CH}_{2}\right)_{8}\right), \delta=31.979$ ppm (a $\left.\mathrm{CH}_{2}\right), \quad \delta=110.00-145.131$ ppm (aromatic benzene carbons), $\delta=155.975$ ppm $(\mathrm{C}=\mathrm{N})$; Anal. calcd. for $\mathrm{C}_{24} \mathrm{H}_{31} \mathrm{CIN}_{4} \mathrm{O}_{4}$ :
C, 60.63; H, 6.53; N, 11.79. Found: C, $60.59 ; \mathrm{H}, 6.51 ; \mathrm{N}, 11.82 ; \mathrm{MS}(\mathrm{m} / \mathrm{z})$ : 99, $236,401,475\left(\mathrm{M}^{+}\right)$

h5 (Cyclopropyl phenyl ketone 2,4dinitrophenylhydrazone): Bright orange solid; yield: $153 \mathrm{mg}$, (47 \%); mp: 190-191 ${ }^{\circ} \mathrm{C}$; IR (ATR): $\bar{u}=3301,3117,2926,2859$, 2292, 2121, 1625, 1587, 1483, 1402, $1322,1263,1117,853,802 \mathrm{~cm}^{-1} ;{ }^{1} \mathrm{H}-\mathrm{NMR}$ (500 $\left.\mathrm{MHz}_{1} \mathrm{CDCl}_{3}\right): \delta=0.69 \mathrm{ppm}(\mathrm{d}, 2 \mathrm{H}$, $\left.\mathrm{J}=5.37, \mathrm{CH}_{2}\right), \delta=0.89 \mathrm{ppm}$ (t(ddd), J=5.37, $6.34,4.89,3.41,7.81,1 \mathrm{H},(\mathrm{E}) \mathrm{CH}), \delta=$ $1.33 \mathrm{ppm}\left(\mathrm{d}, \mathrm{t}, \mathrm{J}=5.86,4.88,2 \mathrm{H}, \mathrm{CH}_{2}\right)$, $\delta=1.73 \mathrm{ppm}(t, 1 \mathrm{H}, \mathrm{J}=6.35,1.95,5.85$, 2.44, (Z) $\mathrm{CH}), \quad \delta=7.18-7.52 \mathrm{ppm}, \mathrm{J}=6.83$ (cyclopropyl substituted aromatic protons), $\delta=7.82-8.27$ ppm, J=9.25, 11.71, 11.76 $\left(\mathrm{NO}_{2}\right.$ substituted aromatic protons), $\delta=9.09$ ppm (s, 1H, (E) NH), $\delta=12.04 \mathrm{ppm}(\mathrm{s}, 1 \mathrm{H}$, (Z) $\mathrm{NH}) .{ }^{13} \mathrm{C}-\mathrm{NMR}\left(500 \mathrm{MHz}, \mathrm{CDCl}_{3}\right)$ : $\delta=76.78 \mathrm{ppm}\left(\mathrm{CH}_{2}\right), \delta=22.04 \mathrm{ppm}\left(\mathrm{CH}_{2}\right)$, $\delta=77.30 \mathrm{ppm}(\mathrm{CH}), \delta=123.56-132.55 \mathrm{ppm}$ (cyclopropyl substituted aromatic carbons) $\delta=136.23-144.63 \mathrm{ppm} \quad\left(\mathrm{NO}_{2}\right.$ substituted aromatic benzene carbons), $\delta=155.65 \mathrm{ppm}$ $(\mathrm{C}=\mathrm{N})$; Anal. calcd. for $\mathrm{C}_{16} \mathrm{H}_{14} \mathrm{~N}_{4} \mathrm{O}_{4}$ : C, 58.90; H, 4.29; N, 17.18. Found: C, 58.92; $\mathrm{H}, 4.35$; $\mathrm{N}, 17.13$; $\mathrm{MS}(\mathrm{m} / \mathrm{z}): 115,177$, $232,278,309,327\left(\mathrm{M}^{+}\right)$

s1 (Phenyl undecyl ketone semicarbazone): Creamy-white colored solid; yield: $193 \mathrm{mg}$, (61 \%); mp: 98.5-99 ${ }^{\circ} \mathrm{C}$; IR (ATR): $\bar{u}=3470,3349,3262,3057$, 2953, 2849, 1681, 1578, 1462, 1377, $1261,1233,1208,968,720,688,517 \mathrm{~cm}^{-}$ ${ }^{1} ;{ }^{1} \mathrm{H}-\mathrm{NMR}(500 \mathrm{MHz}, \mathrm{DMSO}): \delta=0.834 \mathrm{ppm}$ $\left(\mathrm{t}, 3 \mathrm{H}, \mathrm{J}=6.83, \mathrm{CH}_{3}\right), \delta=1.122-1.355 \mathrm{ppm}$ $\left(\mathrm{m}, 16 \mathrm{H}, \mathrm{J}=7.32,\left(\mathrm{CH}_{2}\right)_{8}\right), \delta=1.371 \mathrm{ppm}(\mathrm{t}$, $2 \mathrm{H}, \mathrm{J}=7.809,4.88$, (E) a $\left.\mathrm{CH}_{2}\right) \delta=2.697 \mathrm{ppm}$ $\left(\mathrm{t}, 2 \mathrm{H}, \mathrm{J}=6.83,8.29\right.$, (Z) a $\left.\mathrm{CH}_{2}\right), \quad \delta=6.420$ ppm (s, 2H, NH 2$), \delta=7.306-7.797$ ppm, $\mathrm{J}=7.32,2.44,2.93,1.46(\mathrm{~m}, \mathrm{~m}$ benzene ring protons), $\delta=9.453 \mathrm{ppm}(\mathrm{s}, 1 \mathrm{H}, \mathrm{NH})$ ${ }^{13} \mathrm{C}-\mathrm{NMR}$ (500 MHz, DMSO): $\delta=12.633 \mathrm{ppm}$ $\left(\mathrm{CH}_{3}\right), \quad \delta=22.776-29.706 \mathrm{ppm}\left(\left(\mathrm{CH}_{2}\right)_{8}\right)$, $\delta=31.983$ ppm (a $\left.\mathrm{CH}_{2}\right), \quad \delta=126.706$ $138.193 \mathrm{ppm}$ (aromatic benzene carbons), $\delta=147.942 \mathrm{ppm}(\mathrm{C}=\mathrm{N}), \quad \delta=157.966 \mathrm{ppm}$ $(\mathrm{C}=\mathrm{O})$; Anal. calcd. for $\mathrm{C}_{20} \mathrm{H}_{32} \mathrm{~N}_{2} \mathrm{O}$ : C, 75.95; $\mathrm{H}, 10.13 ; \mathrm{N}, 8.86$. Found: $\mathrm{C}, 75.98$; $\mathrm{H}, 10.17 ; \mathrm{N}, 8.81$; MS (m/z):180, 239, 287, $317\left(\mathrm{M}^{+}\right)$

s2 (Tolyl undecyl ketone semicarbazone): Gelly lemon yellow colored solid; yield: $234 \mathrm{mg}$, (71 \%); $\mathrm{mp}: 101-103{ }^{\circ} \mathrm{C} ;$ FT-IR (ATR): $\overline{\mathrm{u}}=3470$, $3185,2920,2850,1680,1656,1572$, 1457, 1325, 1186, $1097 \mathrm{~cm}^{-1} ;{ }^{1} \mathrm{H}-\mathrm{NMR}$ (500 $\left.\mathrm{MHz} \mathrm{CDCl}_{3}\right): \delta=0.92 \mathrm{ppm}(\mathrm{t}, 3 \mathrm{H}, 5.37$, $4.88,1.95,1.96$, terminal $\left.\mathrm{CH}_{3}\right), \delta=1.213$ - 
$1.328 \mathrm{ppm}(\mathrm{m}, 16 \mathrm{H}, \mathrm{J}=3.9,13.18,6.83$, $\left.6.84,7.32,5.37,\left(\mathrm{CH}_{2}\right)_{8}\right), \delta=1.38 \mathrm{ppm}(\mathrm{p}, 2$ $\mathrm{H}, \mathrm{J}=6.34,7.32,7.81,7.32,6.83$, (E) $\beta$ $\left.\mathrm{CH}_{2}\right), \delta=1.55 \mathrm{ppm}(\mathrm{p}, 2 \mathrm{H}, \mathrm{J}=5.86,5.37$, 5.37, (Z) $\left.\beta \mathrm{CH}_{2}\right), \delta=1.63 \mathrm{ppm}\left(\mathrm{s}, 2 \mathrm{H}, \mathrm{NH}_{2}\right)$, $\delta=2.39 \mathrm{ppm}\left(\mathrm{s}, 3 \mathrm{H}\right.$, tolyl $\left.\mathrm{CH}_{3}\right), \delta=2.59 \mathrm{ppm}$ $\left(\mathrm{t}, 2 \mathrm{H}, \mathrm{J}=8.3,7.81\right.$, (E) a $\left.\mathrm{CH}_{2}\right), \delta=2.93$ ppm ( $t, 2 \mathrm{H}, \mathrm{J}=7.81,7.32$, (Z) a $\left.\mathrm{CH}_{2}\right)$, , $\delta=7.57 \mathrm{ppm}(\mathrm{dd}, \mathrm{J}=1.95,1.46,6.83,2 \mathrm{H}$ aromatic protons), $\delta=7.81 \mathrm{ppm}(\mathrm{d}, \mathrm{J}=6.34$, $1 \mathrm{H}$ aromatic proton), $\delta=7.87 \mathrm{ppm}$ (d, $\mathrm{J}=4.88,1 \mathrm{H}$ aromatic proton), $\delta=7.94 \mathrm{ppm}$ $(\mathrm{s}, 1 \mathrm{H}, \mathrm{NH}){ }^{13} \mathrm{C}-\mathrm{NMR}\left(500 \mathrm{MHz} \mathrm{CDCl}_{3}\right)$ : $\delta=12.13 \mathrm{ppm}$ (terminal $\left.\mathrm{CH}_{3}\right), \quad \delta=22.67$ $29.91 \mathrm{ppm}\left(\left(\mathrm{CH}_{2}\right)_{8}\right), \delta=31.92 \mathrm{ppm}\left(\mathrm{a} \mathrm{CH}_{2}\right)$, $\delta=38.55$ ppm (tolyl $\left.\mathrm{CH}_{3}\right), \delta=126.04-199.93$ ppm (aromatic benzene carbons), $\delta=200.36$ ppm $(\mathrm{C}=\mathrm{N}), \delta=222.775 \mathrm{ppm}(\mathrm{C}=\mathrm{O}) ; 822$. Anal. calcd. for $\mathrm{C}_{21} \mathrm{H}_{34} \mathrm{~N}_{2} \mathrm{O}$ : C, 76.36; $\mathrm{H}$, 10.30; N, 8.48. Found: C, 76.35; H, 10.34; $\mathrm{N}, 8.43$; MS (m/z): 258, 287, 300, 331 $\left(\mathrm{M}^{+}\right)$

\section{s3 (Bromophenyl undecyl ketone} semicarbazone): White solid; yield: 288 mg, (73 \%); mp: $120-121^{\circ} \mathrm{C}$; FT-IR (ATR): $\bar{u}=3469,3260,3133,2921,2851,1682$, 1577, 1458, 1406, 1314, 1093, 987, 913 $\mathrm{cm}^{-1} ;{ }^{1} \mathrm{H}-\mathrm{NMR}\left(500 \mathrm{MHz} \mathrm{CDCl}_{3}\right): \delta=0.796$ ppm (t, 3H, J=6.83, $\left.\mathrm{CH}_{3}\right), \delta=0.796-1.186$ ppm $\left(\mathrm{m}, 16 \mathrm{H},\left(\mathrm{CH}_{2}\right)_{8}\right), \delta=1.296 \mathrm{ppm}(\mathrm{p}$, $2 \mathrm{H}, \mathrm{J}=7.32,7.75,7.81$, (E) $\left.\beta \mathrm{CH}_{2}\right)$, $\delta=1.424 \mathrm{ppm}(\mathrm{t}, 2 \mathrm{H}, \mathrm{J}=7.81,8.29$, (E) a $\left.\mathrm{CH}_{2}\right) \delta=1.565 \mathrm{ppm}\left(\mathrm{s}, 2 \mathrm{H},(\mathrm{Z}) \beta \mathrm{CH}_{2}\right)$, $\delta=2.268 \mathrm{ppm}\left(\mathrm{s}, 2 \mathrm{H},(\mathrm{Z})\right.$ a $\left.\mathrm{CH}_{2}\right), \delta=2.541$ ppm ( $\left.t, 2 \mathrm{H}, \mathrm{J}=8.3, \mathrm{NH}_{2}\right), \delta=7.400 \mathrm{ppm}$ (dd, dd, J=2.44, 4.40, 7.32, 2.44 aromatic protons, $4 \mathrm{H}), \delta=8.972 \mathrm{ppm}(\mathrm{s}, 1 \mathrm{H}, \mathrm{NH}){ }^{13} \mathrm{C}-$ NMR $\left(500 \mathrm{MHz}, \mathrm{CDCl}_{3}\right): \delta=13.086 \mathrm{ppm}$ $\left(\mathrm{CH}_{3}\right), \quad \delta=21.675-28.743 \mathrm{ppm} \quad\left(\left(\mathrm{CH}_{2}\right)_{8}\right)$, $\delta=30.913$ ppm (a $\left.\mathrm{CH}_{2}\right), \quad \delta=122.489$ $148.726 \mathrm{ppm}$ (aromatic benzene carbons), $\delta=157.300 \mathrm{ppm}(\mathrm{C}=\mathrm{N}), \quad \delta=176.950 \mathrm{ppm}$ $(\mathrm{C}=\mathrm{O})$; Anal. calcd. for $\mathrm{C}_{20} \mathrm{H}_{31} \mathrm{BrN}_{2} \mathrm{O}$ : C, 60.76; $\mathrm{H}, 7.85 ; \mathrm{N}, 7.09$. Found: C, 60.71; $\mathrm{H}, 7.88 ; \mathrm{N}, 7.03 \mathrm{MS}(\mathrm{m} / \mathrm{z}): 232,303,334$, $352,396\left(\mathrm{M}^{+}\right)$

s4 (Chlorophenyl undecyl ketone semicarbazone): Creamish white solid; yield: $259 \mathrm{mg}$, (74 \%); mp: 106-107 ${ }^{\circ} \mathrm{C}$; FT-IR (ATR): $\bar{u}=3533,3485,3416,3174$, 3089, 2919, 2849, 1696, 1605, 1553, $1463,1422,1317,1093,1146,1010,834$ $\mathrm{cm}^{-1} ;{ }^{1} \mathrm{H}-\mathrm{NMR}$ (500 MHz, DMSO): $\delta=0.829$ ppm $\left(\mathrm{t}, 3 \mathrm{H}, \mathrm{J}=7.32,6.83, \mathrm{CH}_{3}\right), \delta=1.212-$ $1.330 \mathrm{ppm}\left(\mathrm{m}, 16 \mathrm{H},\left(\mathrm{CH}_{2}\right)_{8}\right), \delta=1.258 \mathrm{ppm}$ $\left(p, 2 \mathrm{H},(E) \beta \mathrm{CH}_{2}\right), \delta=1.330 \mathrm{ppm}(\mathrm{t}, 2 \mathrm{H},(\mathrm{E})$ a $\left.\mathrm{CH}_{2}\right), \delta=2.154 \mathrm{ppm}\left(\mathrm{p}, 2 \mathrm{H},(\mathrm{Z}) \beta \mathrm{CH}_{2}\right)$, $\delta=2.701 \mathrm{ppm}(\mathrm{t}, 2 \mathrm{H}, \mathrm{J}=6.34,7.81$ (Z) a $\left.\mathrm{CH}_{2}\right), \delta=6.471 \mathrm{ppm}\left(\mathrm{s}, 2 \mathrm{H}, \mathrm{NH}_{2}\right), \delta=7.376-$ $7.837 \mathrm{ppm}(\mathrm{dd}, \mathrm{dd}, \mathrm{J}=1.95,8.78$, aromatic protons, $4 \mathrm{H}), \delta=9.526 \mathrm{ppm}(\mathrm{s}, 1 \mathrm{H}, \mathrm{NH})$
${ }^{13} \mathrm{C}-\mathrm{NMR}(500 \mathrm{MHz}, \mathrm{DMSO}): \delta=14.625 \mathrm{ppm}$ $\left(\mathrm{CH}_{3}\right), \quad \delta=22.780-29.714$ ppm $\left(\left(\mathrm{CH}_{2}\right)_{8}\right)$, $\delta=31.987$ ppm (a $\left.\mathrm{CH}_{2}\right), \quad \delta=128.525-$ $137.034 \mathrm{ppm}$ (aromatic benzene carbons), $\delta=146.703 \mathrm{ppm}(\mathrm{C}=\mathrm{N}), \quad \delta=157.893 \mathrm{ppm}$ $(\mathrm{C}=\mathrm{O})$; Anal. calcd. for $\mathrm{C}_{20} \mathrm{H}_{31} \mathrm{~N}_{2} \mathrm{O}$ : C, 68.38; H, 8.83; N, 7.98. Found: C, 68.42; $\mathrm{H}, 8.85 ; \mathrm{N}, 7.91 ; \mathrm{MS}(\mathrm{m} / \mathrm{z}): 170,264,286$, $329,351\left(\mathrm{M}^{+}\right)$

s5 (Cyclopropyl phenyl ketone semicarbazone): White solid; yield: 119 mg, (53 \%); mp: $169-170^{\circ} \mathrm{C}$; FT-IR (ATR): $\overline{\mathrm{u}}=3372,3342,3273,3216,3005,1665$, $1596,1466,1400,1363,1298,1147$, 1086, 1036, 966, $930 \mathrm{~cm}^{-1} ;{ }^{1} \mathrm{H}-\mathrm{NMR}$ (500 $\left.\mathrm{MHz} \mathrm{CDCl}_{3}\right): \delta=0.62 \mathrm{ppm}(\mathrm{dt}, \mathrm{J}=1.5,3,4$, $\left.2 \mathrm{H}, \mathrm{CH}_{2}\right), \delta=1.136 \mathrm{ppm}(\mathrm{td}, 2 \mathrm{H}, \mathrm{J}=4,1.5$, $\left.\mathrm{CH}_{2}\right), \delta=1.57 \mathrm{ppm}(\mathrm{m}, \mathrm{H}, \mathrm{CH}), \delta=1.78$ ppm (s, $\left.2 \mathrm{H}, \mathrm{NH}_{2}\right), \delta=7.72 \mathrm{ppm}$ (aromatics, $\mathrm{J}=6.83,2.44,1.46,5.86,5 \mathrm{H}), \delta=8.53 \mathrm{ppm}$ (s, $1 \mathrm{H}, \mathrm{NH}) .{ }^{13} \mathrm{C}-\mathrm{NMR}\left(500 \mathrm{MHz} \mathrm{CDCl}_{3}\right)$ : $\delta=76.79 \mathrm{ppm}\left(\mathrm{CH}_{2}\right), \delta=77.04 \mathrm{ppm}\left(\mathrm{CH}_{2}\right)$, $\delta=77.30 \mathrm{ppm}(\mathrm{CH}), \delta=127.10-136.70 \mathrm{ppm}$ (aromatic carbons), $\delta=148.84 \mathrm{ppm}(\mathrm{C}=\mathrm{N})$, $\delta=156.78 \mathrm{ppm}(\mathrm{C}=\mathrm{O})$; Anal. calcd. for $\mathrm{C}_{12} \mathrm{H}_{14} \mathrm{~N}_{2} \mathrm{O}: \mathrm{C}, 71.29 ; \mathrm{H}, 6.93 ; \mathrm{N}, 13.86$. Found: $\mathrm{C}, 71.31 ; \mathrm{H}, 6.96 ; \mathrm{N}, 13.82 ; \mathrm{MS}$ $(\mathrm{m} / \mathrm{z}): 130,159,173,203\left(\mathrm{M}^{+}\right)$

p1 (1-(2,4-dinitrophenyl)-3,5dimethyl-1H pyrazole): (11) Brown colored solid; yield: $165 \mathrm{mg}$, (63 \%); mp: 105-106 ${ }^{\circ} \mathrm{C}$; FT-IR (ATR): $\bar{u}=3080,2993$, $1630,1608,1522,1381 \mathrm{~cm}^{-1} ; 1 \mathrm{H}-\mathrm{NMR}(500$ $\left.\mathrm{MHz}_{1} \mathrm{CDCl}_{3}\right): \delta=2.15 \mathrm{ppm}\left(\mathrm{d}, 3 \mathrm{H}, \mathrm{CH}_{3}\right)$, $\delta=2.72 \mathrm{ppm}\left(\mathrm{d}, 3 \mathrm{H}, \mathrm{CH}_{3}\right), \delta=6.00 \mathrm{ppm}(\mathrm{s}$, $1 \mathrm{H}, \mathrm{CH}), \delta=7.66 \mathrm{ppm}(\mathrm{d}, 1 \mathrm{H}, \mathrm{J}=8.29$, aromatic), $\delta=7.76 \mathrm{ppm}(\mathrm{s}, 1 \mathrm{H}$, aromatic), $\delta=8.45 \mathrm{ppm}$ (d, $1 \mathrm{H}, \mathrm{J}=2.44$, aromatic), $\delta=8.50 \mathrm{ppm}$ (d, $1 \mathrm{H}, \mathrm{J}=1.95$, aromatic), $\delta=8.68 \mathrm{ppm}$ (d, aromatics, $\mathrm{d}=2.44,{ }^{13} \mathrm{C}-$ NMR $\left(500 \mathrm{MHz}, \mathrm{CDCl}_{3}\right): \delta=31.34 \mathrm{ppm}$ $\left(\mathrm{CH}_{3}\right), \delta=36.19 \mathrm{ppm}\left(\mathrm{CH}_{3}\right), \delta=108.80 \mathrm{ppm}$ $(\mathrm{CH}), \quad \delta=120.88-146.11 \mathrm{ppm}$ (aromatic carbons), $\delta=152.09 \mathrm{ppm}(\mathrm{C}-\mathrm{N}), \delta=162.57$ ppm $(\mathrm{C}=\mathrm{N})$; Anal. calcd. for $\mathrm{C}_{11} \mathrm{H}_{10} \mathrm{~N}_{4} \mathrm{O}$ : C, 50.38; $\mathrm{H}, 3.82 ; \mathrm{N}, 21.37$. Found: $\mathrm{C}, 66.04$; $\mathrm{H}, 7.46 ; \mathrm{N}, 12.37$; $\mathrm{MS}(\mathrm{m} / \mathrm{z}): 95,102,169$, $186,199,232,264\left(M^{+}+1\right)$

\section{Antimicrobial activity}

Antimicrobial activity against Pseudomonas aeruginosa ATCC 27853, Escherichia coli ATCC 25922, Klebsiella pneumoniae ATCC 4352, Proteus mirabilis ATCC 14153, Enterococcus faecalis ATCC 29212, Staphylococcus epidermidis ATCC 12228, Staphylococcus aureus ATCC 29213, Candida albicans ATCC 10231, Candida parapsilosis ATCC 22019, Candida tropicalis ATCC 750 was determined by the microbroth dilution method according to the 
recommendations of Clinical Laboratory Standarts Institute (CLSI). Mueller Hinton broth (Difco, Detroid, USA) was used for bacterial species and RPMI- 1640 (Sigma) was used for Candida species throughout the experiments. Serial two fold dilutions ranging from 5000 to $1.22 \mu \mathrm{L}$ were prepared in the medium. The inoculum was prepared using a 4-6 $\mathrm{h}$ broth culture of each bacterial type and $24 \mathrm{~h}$ culture of yeast strains adjusted to a turbidity equivalent to 0.5 McFarland Standard, diluted in broth media to give a final concentration of $5 \times 10^{5}$ $\mathrm{cfu} / \mathrm{mL}$ for bacteria and $5 \times 10^{3} \mathrm{cfu} / \mathrm{mL}$ for

\section{RESULTS AND DISCUSSION}

\section{Chemistry}

Novel long chain alkyl, phenyl and cyclopropyl containing semicarbazones (s1s5) and 2,4-dinitrophenylhydrazones (h1h5) were synthesized in this study, with the aim to investigate their anitimicrobial activities. Besides, a pyrazole ring carrying 2,4-dinitrophenylhydrazone p1(1-(2,4dinitrophenyl)-3,5-dimethyl-1H pyrazole) was synthesized from the reaction of 2,4dinitrophenylhydrazine with a diketone acetylacetone.

The semicarbazones and hydrazones were obtained from their corresponding ketones (k1-k5) prepared by Friedel-Crafts yeast in the test tray. The trays were covered and placed into plastic bags to prevent evaporation. Microplates were incubated for $18-24 \mathrm{~h}$ at $35^{\circ} \mathrm{C}$ for bacteria and $46-50 \mathrm{~h}$ at $35^{\circ} \mathrm{C}$ for yeast. The MIC value was evaluated as the lowest concentration of the compound that the visible proliferation has not occurred. Ciprofloxacin and fluconazole were included throughout the experiments in the study as standard antimicrobials for bacteria and fungi. The results for both antimicrobials were found according to the CLSI criteria. (14-15)

acylation. The substances (h1-h5) (s1-s5) and (p1) (Table 5) were checked with spectroscopic methods. Their $E / Z$ isomerization was $1: 1$ as detected by their NMR spectra. The signals of isomer protons of the double bond resonated on different chemical shifts with same magnitude. Therefore the ratio of isomers was determined as $50 \%$. $\mathrm{E}$ and $\mathrm{Z}$ isomers had different shielding effects in ${ }^{1} \mathrm{H}-\mathrm{NMR}$ spectrum depending on the electronic densities in the molecule due to the location of the substituents. These were seen and determined in the upper or lower fields according to the shielding effects. (16) $\mathrm{C}=\mathrm{N}$ groups of synthesized semicarbazones and hydrazones showed peaks around $1550 \mathrm{~cm}^{-}$ 1 in the FT-IR spectra.

Table 5 Structures of the synthesized hydrazones, semicarbazones and pyrazole.

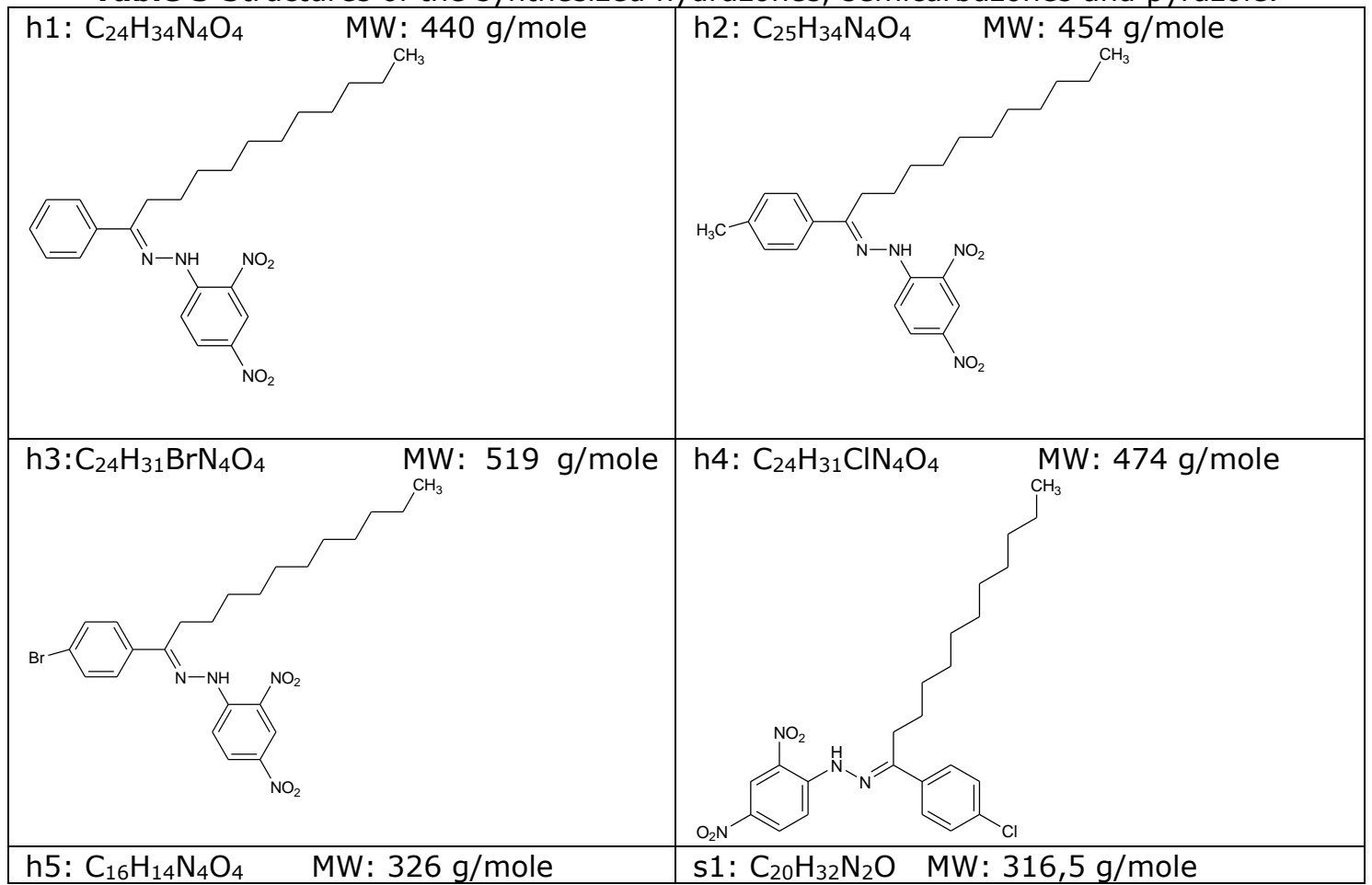




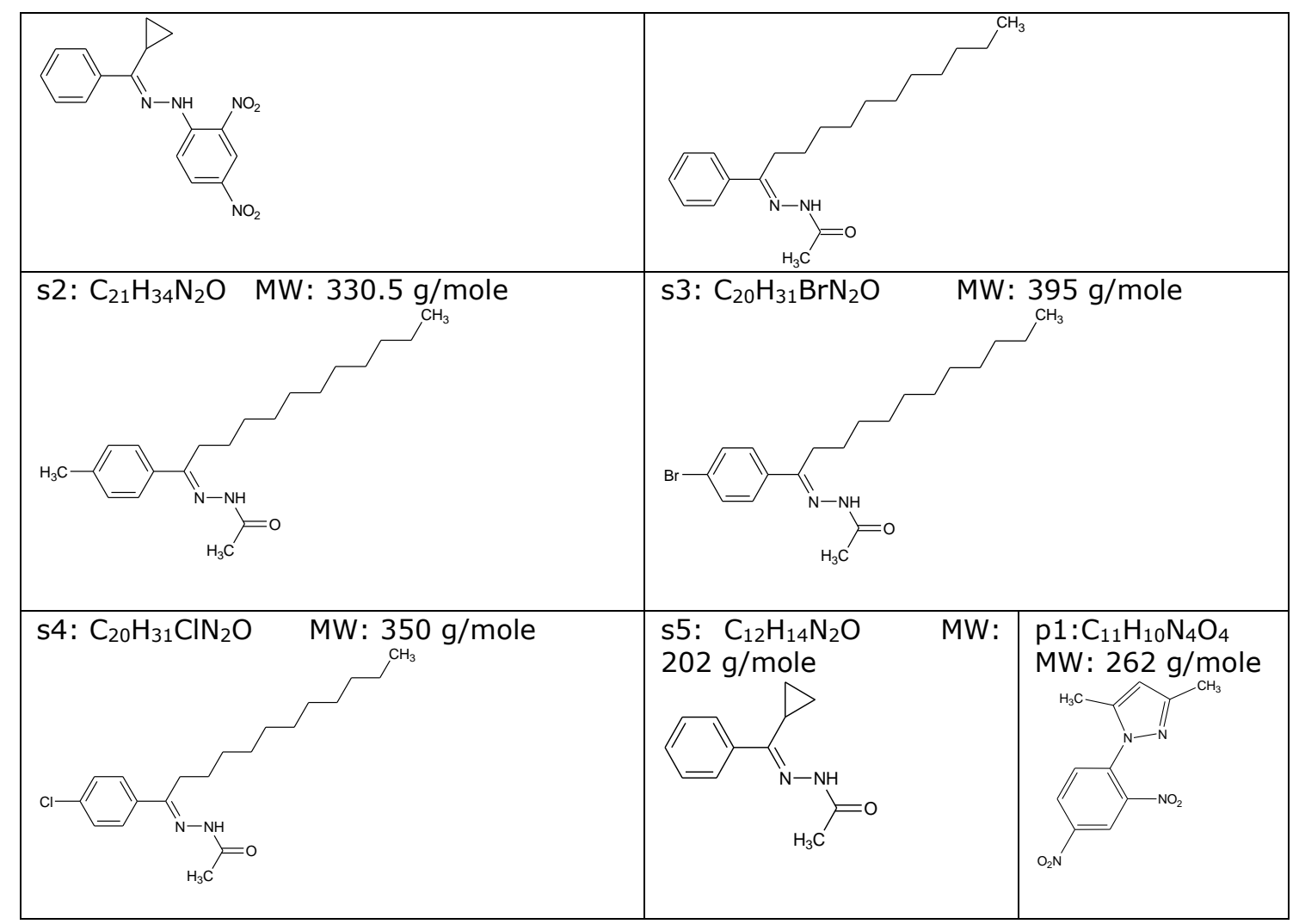

\section{Antimicrobial activities}

The structure-antimicrobial activity relationship in this study was explained due to the results of the antimicrobial studies summarized in Table 6.

Cyclopropyl and nitro groups were effective in antimicrobial activity. This cyclopropyl and two deactivating nitro groups increased the activity of h5. Nitro groups with negative charged oxygen atom accelerated the attack of $\mathbf{h} \mathbf{5}$ against three bacterial and two fungal species. Methyl groups were also effective by making $\mathrm{H}$-bridges with bacteria according to their hyperconjugative character. Methyl and nucleophilic bromo groups had diminished the population of bacteria and fungi. According to the obtained antimicrobial results (Table 6) synthesized hydrazones (h1 -h5) were more antimicrobial active than that of semicarbazones (s1- s5). Pyrazole was the most effective one. Hydrazones in this study have more phenyl ring than that of semicarbazones. 2,4-dinitro phenyl substituent is more effective than $\mathrm{CO}-\mathrm{NH}_{2}$ group, therefore h1>s1. Nitro group with two oxygens had played an important role here. Alkyl and unsubstituted phenyl ring had no effect, as seen with s1. s1 showed no antimicrobial activity. 2,4-dinitro phenyl substituent made hydrazone h1 only against one bacterial species. The activity range of semicarbazones is as follows; s5>s2>s3>s4 $\geq s 1 . ~ s 5$ is the best one because it carries a cyclopropyl substituent instead of a long alkyl chain. ring. s5 has two rings, one non-aromatic cyclopropyl ring and one aromatic phenyl ring. These ring configurations made $\mathbf{s 5}$ the most effective substance. $\mathbf{5 5}$ was effective one among the semicarbazones. s5 was effective against four bacterial species. h1 with long alkyl chain showed no activity. h5 with phenyl, cyclopropyl and 2,4dinitrophenyl substituents were more effective than $\mathbf{5 5}$. h5 was active against three bacterial and two fungal species. The data of Table 6 supported that the activity range of the hydrazones h1-h5 followed as h5 $\geq h 2>h 3>h 4>h 1$. h5 with cyclopropyl ring was active against three bacteria and two fungi species. Tolyl group led $\mathbf{h} \mathbf{2}$ to be equally active with $\mathbf{h} \mathbf{5}$ only against five bacterial species. Long chain alkyl group of h2 was a deactivating group but tolyl with electron donating $\mathrm{CH}_{3}$ group activated $\mathbf{h} \mathbf{2}$ in the range of $\mathbf{h} \mathbf{2}>\mathbf{h} \mathbf{3}>\mathbf{h} \mathbf{4}>\mathbf{h} \mathbf{1}$. Electron donating groups like $\mathrm{CH}_{3}$ increased the activity and electron-withdrawing groups like bromo and chloro decreased the activity. No substituent carrying phenyl ring was the most uneffective one obtained with h1. The substance p1 with heterocyclic pyrazole character was the most active against six bacteria and three fungi species. Heterocyclic pyrazole group showed an 
importance in being a good antimicrobial agent.

\section{CONCLUSION}

The synthesized novel hydrazones were found to be better antimicrobial agents than synthesized semicarbazones. Their preparation is cheap and environmentally friendly. Among them, h5 and s5 were the best inhibitory substances against the mentioned species of bacteria and fungi. p1 (1-(2,4-dinitrophenyl)-3,5-dimethyl-1H pyrazole) was proposed to be a good general antimicrobial source with its wide effective spectrum. Hydrazones are more stable than semicarbazones by more electron delocalization. This resonance ability made the hydrazones more active.
According to the literature report lipophilic long alkyl chain with its $\mathrm{sp}^{3}$ hybridization decreased the activity (17). Substitutions of hydrazones and semicarbazones' structures were also effective due to the results obtained in Table 6. Cyclopropyl's $\mathrm{sp}^{2}$ increased the activity as seen with h5-h1 and s5-s1. Substitutions on phenyl ring were important, too. (18-21) Electron donating group increased the activity as obtained with h2>h1 and s2>s1. Electron withdrawing groups decreased the activity as seen with $\mathbf{h} \mathbf{3}>\mathbf{h} \mathbf{4}>\mathbf{h} \mathbf{1}$ and $\mathbf{s} \mathbf{3} \mathbf{s} \mathbf{4} \mathbf{s} \mathbf{1}$. Phenyl ring with none substitution was the least active structure as $\mathbf{h} \mathbf{1}$ and s1. p1 with 2,4-dinitrophenyl and two methyl groups on pyrazole ring was the best resonance stable compound and therefore exhibited the best activity. 
Table 6 Antimicrobial activities of hydrazones, semicarbazones and pyrazole.

\begin{tabular}{|c|c|c|c|c|c|c|c|c|c|c|}
\hline & $\begin{array}{l}\text { P. aeruginosa } \\
\text { ATCC } 27853\end{array}$ & $\begin{array}{l}\text { E. coli } \\
\text { ATCC } \\
25922\end{array}$ & $\begin{array}{l}\text { K. pneumoniae } \\
\text { ATCC } 4352\end{array}$ & $\begin{array}{l}\text { P. } \quad \text { mirabilis } \\
\text { ATCC } 14153\end{array}$ & $\begin{array}{l}\text { S. aureus } \\
\text { ATCC } \\
29213\end{array}$ & $\begin{array}{l}\text { S. epidermidis } \\
\text { ATCC } 12228\end{array}$ & $\begin{array}{l}\text { E. } \\
\text { faecalis } \\
\text { ATCC } \\
29212\end{array}$ & $\begin{array}{l}\text { C. } \\
\text { albicans } \\
\text { ATCC } \\
10231\end{array}$ & $\begin{array}{l}\text { C. parapsilosis } \\
\text { ATCC } 22019\end{array}$ & $\begin{array}{l}\text { C. } \\
\text { tropicalis } \\
\text { ATCC } \\
750\end{array}$ \\
\hline s1 & - & - & - & - & - & - & - & - & - & - \\
\hline s2 & - & 312.5 & - & 625 & - & - & - & - & - & - \\
\hline s3 & - & - & - & - & 625 & - & 1250 & - & - & - \\
\hline s4 & - & - & - & - & - & - & - & - & - & - \\
\hline s5 & - & 312.5 & 625 & 625 & - & 1250 & - & - & - & - \\
\hline h1 & - & - & - & - & - & - & 625 & - & - & - \\
\hline h2 & 312.5 & 625 & - & - & 1250 & 1250 & 625 & - & - & - \\
\hline h3 & - & - & 625 & 625 & - & - & 625 & 78.12 & - & - \\
\hline h4 & - & 625 & - & - & - & - & 625 & - & - & - \\
\hline h5 & - & - & - & - & 1250 & 1250 & 625 & - & 312.5 & 312.5 \\
\hline p1 & - & 625 & 312.5 & 625 & 2.44 & 1.22 & 312.5 & 4.88 & 39.06 & 19.53 \\
\hline
\end{tabular}




\section{ACKNOWLEDGEMENTS}

This study was supported by the Istanbul University Scientific Research Projects Division with the project number of 20665.

\section{REFERENCES}

1. Reddy KH, Reddy PS, Babu PR, (2000) Nuclease activity of 2-substituted heteroaromatic thiosemicarbazone and semicarbazone copper (II) complexes.Transition Metal Chemistry 25, 154-160.

2. Babahan I, Coban EP, Biyik H, (2013) Synthesis, characterization and antimicrobial activities of vic-dioxime derivatives containing heteroaromatic hydrazine groups and their metal complexes.Maejo International Journal of Science and Technology 7:01, 26-41.

3. Enyedy EA, Bognar GM, Nagy NV, Jakusch T, Kiss T, (2014) Solution speciation of potential anticancer metal complexes of salicylaldehyde semicarbazone and its bromo derivative.Polyhedron 67, 242-252.

4. Kiasat AR, Kazemi F, Mehrjardi MF, (2007) Basic alumina as an efficient catalyst for preparation of semicarbazones in solvent free conditions. Journal of Chinese Chemical Society 54, 1337-1339.

5. Sameem S, Kumar N, Pathak D, (2012) Synthesis and anticonvulsant activity of some newer semicarbazone derivatives. International Journal of Pharmaceutical Sciences and Drug Research 4:3, 195-198.

6. Gup R, (2013), Synthesis and spectroscopic studies of new bisacylhydrazone compounds. Chimica acta Turcica 35:1, 55-61.

7. Torje IA, Valean AM, Cristea C, (2012) Phenothiazine-carboxaldehyde-hydrazone derivatives, synthesis, characterisation and electronic properties. Academia Romana 57:4-5, 337-344.

8. Hu J, Chen S, Sun Y, Yang J, Rao Y, (2012) Synthesis of tri-and tetrasubstituted pyrazoles via Ru (II) catalysis: Intramolecular aerobic oxidative $\mathrm{C}-\mathrm{N}$ coupling. Organic Letters 14:19, 50305033.

9. Popiolek L, (2017) Hydrazide-hydrazones as potential antimicrobial agents: Overview of the literature since 2010. Medicinal Chemistry Research 26:2, 287-301.

10. Rahmatzadeh SS, Karami B, Khodabakhshi S, (2015) A modified and practical synthetic route to indazoles and pyrazoles using tungstate sulfuric acid. Journal of the Chinese Chemical Society 62, 17-20.

11. Anandarajagopal $K$, Sunilson JA, Illavarasu A, Thangavelpandian N, Kalijaran R, (2010) Antiepileptic and antimicrobial activities of novel 1(unsubstituted/substituted)-3,5-dimethyl$1 \mathrm{H}$-pyrazole derivatives. International Journal of ChemTech Research 281, 45-49.

12. Parashar B, Jain A, Bharadwaj S, Sharma VK, (2012) Synthesis and pharmacological properties of some novel pyrazolidine and pyrazole derivatives. Medicinal Chemistry Research 21:8, 16921699.

13. Breusch FL, Baykut F, (1953) Darstellung der Di-n-alkyl-ketone mit 15bis 22 C-Atomen (V.Mitteil. über isomere und homologe Reihen)Chemische Berichte 86, 684-688.

14. Clinical and Laboratory Standards Institute, (1997) Reference Method for Broth Dilution Antifungal susceptibility testing of yeasts; Approved Standard- 2rd ed. M 27-A2; CLSI, Wayne, PA.

15. Clinical and Laboratory Standards Institute, (2006) Methods for dilution antimicrobial susceptibility tests for bacteria that grow aerobically, 7th ed. Approved Standard M7-A7; CLSI, Wayne, PA.

16. Balsamo A, Bertini S, Gervasi G, Lapucci A, Nencetti S, Orlandini E, Rapposelli S, Rossello A, Soldani G, (2001) Enantiopure 3-(arylmethylidene)aminoxy-2-

methylpropionic acids: synthesis and antiinflammatory properties. Eur. J. Med. Chem. 36, 799-807.

17. Malik I, Csöllei J, Jampilek J, Stanzel L, Zadrazilova I, Hosek J, Pospisilova S, Cizek A, Coffey A, O'Mahony J, (2016) The Structure-Antimicrobial Activity Relationships of a Promising Class of the Compounds Containing the N-Arylpiperazine Scaffold. Molecules. 21, 1274.

18. Zani $F$, Vicini $P$, Incerti $M$, (2004) Synthesis and Antimicrobial properties of 2(benzylidene-amino)-benzo[d] isothiazol-3- 
ones. European Journal of Medicinal Chemistry. 39, 135-140.

19. Lien EJC, Hansch C, Anderson SM, (1968) Structure-activity correlations for antibacterial agents on gram -positive and gram-negative cells. J. Med. Chem. 11, 430-441.

20. Gopalakrishnan M, Thanusu J, Kanagarajan V, (2009) Design, synthesis, spectral analysis and in vitro microbiological evaluation of 2-phenyl-3-(4,6diarylpyrimidin-2-yl)thiazolidin-4-ones.

Journal of Enzyme Inhibition and Medicinal Chemistry. 24(5), 1088-1094.

21. Pitucha $M$, Pachuta A, Kaczor AA, (2013) New five-membered ring heterocyclic compounds with antibacterial and antifungal activity; Microbial pathogens and strategies for combating them: science, technology and education. (A. MendezVilas, Ed) Formatex, 562-573. 